\title{
Adición de metil- $\beta$-ciclodextrina cargada de colesterol sobre la criopreservación de semen de toros Holstein Friesian
}

\author{
Addition of methyl- $\beta$-cyclodextrin loaded with cholesterol (CLC) on the \\ cryopreservation of semen of Holstein Friesian bulls
}

Hugo Paúl Viñán Díaz', Juan Enrique Paucar Espinoza ${ }^{2,4}$, Enrique Alvarado Malca ${ }^{3}$

\section{Resumen}

Se evaluó el efecto de la adición de metil- $\beta$-ciclodextrina cargada con colesterol (CLC) sobre la criopreservación de semen de toros Holstein Friesian a través de la valoración de las características seminales pre y pos-congelación. Se utilizaron cuatro toros de 3-4 años, obteniéndose 28 eyaculados, que fueron divididos en tres alicouotas, donde se agregó el CLC: 0, 1.5 y 2 mg de CLC por cada 120 millones de espermatozoides (T1, T2 y T3, respectivamente) a $37^{\circ} \mathrm{C}$ y fueron incubados por 15 minutos. Las muestras fueron refrigeradas a $5{ }^{\circ} \mathrm{C}$ durante 18 horas para su estabilización. La congelación del semen se hizo en pajillas de $0.5 \mathrm{ml}$ usando vapores de nitrógeno por 7 minutos y luego sumergidas en nitrógeno líquido. No se encontraron diferencias estadísticas entre tratamientos en todas las características del semen refrigerado. Los resultados pos-descongelación revelaron mejoras significativas en los tratamientos con colesterol, obteniendo una motilidad total del $67.73,79.59$ y $74.73 \%$; integridad de membrana de $46.89,60.43$ y $55.06 \%$ y vitalidad de 63.26, 75.84 y 70.53\% para T1, T2 y T3, respectivamente $(\mathrm{p}<0.05)$ No hubo diferencias significativas entre tratamientos con respecto a las anormalidades morfológicas.

Palabras clave: toro; ciclodextrina; colesterol; criopreservación; semen

\section{Abstract}

The effect of the addition of cholesterol-loaded methyl- $\beta$-cyclodextrin (CLC) on the semen cryopreservation of Holstein Friesian bulls was assessed through the assessment of pre and post-freezing seminal characteristics. Four bulls of 3-4 years of age were used and 28 ejaculates were collected. The ejaculates were divided into three aliquots, where

${ }^{1}$ Facultad de Zootecnia, Universidad Nacional Agraria La Molina, Lima, Perú

${ }^{2}$ Banco Nacional de Semen, Universidad Nacional Agraria La Molina, Lima, Perú

${ }^{3}$ Departamento de Producción Animal, Facultad de Zootecnia, Universidad Nacional Agraria La Molina, Lima, Perú

${ }^{4}$ E-mail: epaucar@lamolina.edu.pe

Recibido: 18 de enero de 2019

Aceptado para publicación: 1 de octubre de 2019 
the CLC was added: $0,1.5$ and $2 \mathrm{mg}$ of CLC per 120 million sperm cells (T1, T2 and T3 respectively) at $37^{\circ} \mathrm{C}$ and incubated for 15 minutes. The samples were refrigerated at $5{ }^{\circ} \mathrm{C}$ for 18 hours for stabilization. Semen was frozen in $0.5 \mathrm{ml}$ straws using nitrogen vapours for 7 minutes and then immersed in liquid nitrogen. No statistical differences were found between treatments in all the characteristics of the refrigerated semen. The post-thawing results revealed significant improvements in the cholesterol treatments, obtaining a total motility of $67.73,79.59$ and $74.73 \%$; membrane integrity of $46.89,60.43$ and $55.06 \%$, and vitality of $63.26,75.84$ and $70.53 \%$ for $\mathrm{T} 1, \mathrm{~T} 2$ and $\mathrm{T} 3$, respectively $(\mathrm{p}<0.05)$. There were no significant differences between treatments with respect to morphological abnormalities.

Key words: bull; cyclodextrin; cholesterol; cryopreservation; semen

\section{INTRODUCCIÓN}

La inseminación artificial (IA) es una técnica que facilita el mejoramiento genético. Según el último censo nacional agropecuario de 2012, se tiene 36982 unidades agropecuarias que utilizan la IA para el manejo reproductivo del ganado (SENAGRO, 2012). La criopreservación es una pieza clave en el procesamiento seminal que permite conservar semen, ovocitos y embriones durante tiempo indefinido. Sin embargo, durante la criopreservación se produce una disminución de la calidad seminal con respecto a la del semen fresco o refrigerado debido al shock térmico, pues se presentan daños bioquímicos y estructurales, especialmente a nivel de membrana plasmática (Hummersted et al., 1990). Estas lesiones provocan la pérdida de componentes intracelulares como enzimas metabólicas y reducción de la motilidad, vitalidad espermática y la capacidad fertilizante del espermatozoide.

El tratamiento con colesterol incorporado a la membrana espermática por medio de ciclodextrinas podría disminuir estos daños, ayudando a preservar la viabilidad del semen pos-descongelación, mejorando la fluidez y estabilidad de la membrana en bajas temperaturas. En vacunos, Purdy y Graham (2004a) observaron diferencias significativas en viabilidad y motilidad entre espermatozoides tratados con ciclodextrinas cargadas de colesterol y sin ellas. Las ciclodextrinas pueden saturarse previamente con colesterol, y van a servir como medio de trasporte para incorporarlo a las membranas plasmáticas (Purdy y Graham, 2004b).

Este trabajo tuvo como objetivo determinar el efecto de la adición de metil- $\beta$ ciclodextrina cargada con colesterol sobre la criopreservación de semen de toros Holstein Friesian, a través de la evaluación de las características seminales pre y pos-congelación del semen (motilidad total y progresiva, vitalidad, anormalidades morfológicas e integridad y funcionalidad de membrana).

\section{Materiales y Métodos}

\section{Lugar del Estudio}

El trabajo de investigación se realizó en las instalaciones del laboratorio del Banco Nacional de Semen de la Universidad Nacional Agraria La Molina (UNALM), ubicado en el distrito de la Molina, Lima-Perú. La zona presenta temperaturas mínimas promedio de $16.2^{\circ} \mathrm{C}$ y máximas de $22.5^{\circ} \mathrm{C}$, humedad relativa promedio anual de $81 \%$ y precipitación anual de $10 \mathrm{~mm}$.

\section{Animales Experimentales}

Se trabajó con cuatro toros jóvenes Holstein Friesian que se encontraban en servicio en el Banco Nacional de Semen. Los 
animales eran de fertilidad comprobada y estaban sujetos a un régimen de colección de semen de una vez por semana. Estaban alojados en corrales independientes, con piso de concreto y tierra, cercos de madera, provisto de sombras, comedero y bebedero, y con adecuada iluminación y ventilación. Todos los animales tenían un régimen de paseo semanal por 60 minutos. La alimentación fue en base de maíz chala (Zea mays) fresco y picado, suplementado con concentrado (14\% proteína, $17.5 \%$ fibra y $68.5 \%$ NDT). El forraje se ofreció dos veces al día y el concentrado por las mañanas.

\section{Ciclodextrinas Cargadas de Colesterol (CLC)}

La solución de CLC fue preparada según lo descrito por Purdy y Graham (2004). En un tubo de ensayo se disolvieron $200 \mathrm{mg}$ de colesterol en $1 \mathrm{ml}$ de cloroformo. En otro tubo de ensayo se diluyó $1 \mathrm{~g}$ de metil- $\beta$ ciclodextrina en $2 \mathrm{ml}$ de metanol. Se adicionó $450 \mu 1$ de la solución de colesterol a la solución de ciclodextrina y se mezcló hasta que la solución estuvo homogénea. En una caja Petri, se removió el solvente usando una corriente de nitrógeno gaseoso. Los cristales resultantes se dejaron secar $24 \mathrm{~h}$, se pasaron a un recipiente de vidrio y se almacenaron a $22{ }^{\circ} \mathrm{C}$. La solución de CLC se preparó agregando $50 \mathrm{mg}$ de CLC a $1 \mathrm{ml}$ de medio de dilución TRIS (300 mM Tris, $94.7 \mathrm{mM}$ de ácido cítrico, $27.75 \mathrm{mM}$ de glucosa y $100 \mathrm{ml}$ de agua desionizada y esterilizada, $\mathrm{pH}$ próximo a 7 y osmolaridad cercana a $330 \mathrm{mOsm} / \mathrm{l}$ ), se mezcló con un vórtex y se incubó en baño maría a $37^{\circ} \mathrm{C}$ hasta el momento de ser utilizado.

\section{Evaluación del Semen}

El semen fue colectado una vez por semana, utilizando un toro como teaser, fijado en una guillotina, bajo sombra, y con piso adecuado para evitar deslizamientos. A los toros, previo a la colección, se les dio un paseo y se les hizo realizar falsas montas. Se utilizó una vagina artificial (IMV, Francia). Los resultados de las evaluaciones del semen se presentan en porcentaje.

\section{Concentración y motilidad espermática}

La concentración espermática y la motilidad fueron determinadas en el módulo de Motilidad y Concentración del Sistema Computarizado (CASA) AndroVision ${ }^{\circledR}$ con microscopio Zeiss AxioScope de Minitube.

\section{Vitalidad espermática}

Para el conteo de los espermatozoides vivos se utilizaron las tinciones de eosina y nigrosina al $2 \%$. Se colocó una gota de $5 \mu 1$ de semen a un extremo de una lámina portaobjeto precalentada a $37^{\circ} \mathrm{C}$ y dos gotas de $5 \mu 1$ de cada colorante. Se homogenizó la gota de semen con la gota de eosina durante $20 \mathrm{~s}$ para que el colorante penetre en los espermatozoides. Luego, esta mezcla se combinó con la nigrosina que sirvió de contraste. Se realizó el frotis y se dejó secar a temperatura ambiente de laboratorio. Las láminas fueron observadas al microscopio con un aumento de 40x.

\section{Anormalidades espermáticas}

Se utilizó la lámina anterior y se observó al microscopio a 400x. Se contabilizaron 200 espermatozoides por conteo y se realizaron tres conteos por muestra.

Funcionalidad de la membrana espermática

Se utilizó la prueba de HOST (Hipo Osmotic Swelling Test), siguiendo el protocolo de Correa y Zavos (1994). Se contaron 200 espermatozoides en el microscopio de contraste de fase a 40X. Se consideraron espermatozoides con membrana funcional $(+)$ los que reaccionaron al estrés hiposmótico mediante la hinchazón de la parte distal de la cola espermática. 


\section{Procesamiento del Semen}

Luego de obtenido el semen se tomó una alícuota para las evaluaciones seminales. El semen fue prediluido (1/1) con el dilutor Andromed ${ }^{\circledR}$ (Minitube). La muestra de semen se dividió en tres alícuotas a las que se agregó a cada tratamiento $0,1.5$ y $2 \mathrm{mg}$ de CLC por cada 120 millones de espermatozoides, se incubaron por $15 \mathrm{~min}$ a temperatura ambiente de laboratorio $\left(22^{\circ} \mathrm{C}\right)$ como describe Purdy y Graham (2004a). Se añadió el dilutor restante para ser envasado con una concentración de $30 \times 10^{6}$ millones de espermatozoides/ml por dosis en pajillas de $0.5 \mathrm{ml}$ (IMV, Francia) y selladas con alcohol polivinílico.

Para la congelación, se hizo un descenso de la temperatura desde $20{ }^{\circ} \mathrm{C}$ hasta $5{ }^{\circ} \mathrm{C}$ en $3 \mathrm{~h}$ y luego se estabilizó durante 18 horas en refrigeradora. La congelación de las pajuelas se hizo por medio de vapores de nitrógeno líquido en 7 min descender a $-140{ }^{\circ} \mathrm{C}$ y luego se sumergieron directamente en el nitrógeno líquido. El descongelamiento de las pajillas se realizó a $38^{\circ} \mathrm{C}$ durante $15-25 \mathrm{~s} \mathrm{y}$ se evaluó la motilidad, vitalidad, anormalidades y funcionalidad de la membrana espermática.

\section{Análisis Estadístico}

Para la determinación del efecto de las CLC se realizó un análisis de diseño en bloques completamente al azar con submuestreos. Para la comparación múltiple de medias se aplicó la prueba de Tukey. Además, fue necesario la transformación arcoseno de los porcentajes de motilidad e integridad de membrana para proceder al análisis estadístico.

Se utilizó el modelo aditivo lineal Yijk = $\mu+T i+B j+E i j+\mathrm{d} i j k$, donde: Yijk $=$ Es la motilidad de la j-ésima repetición al aplicarse el i-ésimo tratamiento de CLC; $\mu=$ Media general; $\mathrm{Ti}=$ Efecto del iésimo tratamiento de CLC; $\mathrm{Bj}=$ Efecto del $\mathrm{j}$-ésimo genotipo; Eij $=$ Error experimental, y dijk $=$ Error del muestreo.

\section{Resultados y Discusión}

\section{Efecto del CLC sobre el Semen Refri- gerado}

No hubo diferencias significativas entre los tratamientos para las variables de evaluación espermática (Cuadro 1), posiblemente a que la adición de CLC solo tiene un efec-

Cuadro 1. Efecto de la adición de ciclodextrinas cargadas de colesterol (CLC) sobre las características microscópicas de semen refrigerado de toros Holstein Friesiar (media \pm error estándar)

\begin{tabular}{lccc}
\hline & $0 \mathrm{mg} \mathrm{CLC}$ & $1.5 \mathrm{mg} \mathrm{CLC}$ & $2 \mathrm{mg}$ CLC \\
& $(\mathrm{T} 1)$ & $(\mathrm{T})$ & $(\mathrm{T})$ \\
\hline Motilidad total (\%) & $92.65 \pm 0.84^{\mathrm{a}}$ & $94.27 \pm 0.94^{\mathrm{a}}$ & $93.67 \pm 0.99^{\mathrm{a}}$ \\
Motilidad progresiva (\%) & $89.74 \pm 0.91^{\mathrm{a}}$ & $91.16 \pm 1.05^{\mathrm{a}}$ & $90.04 \pm 1.07^{\mathrm{a}}$ \\
Vitalidad (\%) & $89.01 \pm 0.65^{\mathrm{a}}$ & $90.85 \pm 0.67^{\mathrm{a}}$ & $90.15 \pm 0.68^{\mathrm{a}}$ \\
Host (\%) & $84.78 \pm 0.67^{\mathrm{a}}$ & $88.14 \pm 0.68^{\mathrm{a}}$ & $86.99 \pm 0.74^{\mathrm{a}}$ \\
Morfología (\%) & $5.04 \pm 0.17^{\mathrm{a}}$ & $4.57 \pm 0.17^{\mathrm{a}}$ & $4.86 \pm 0.18^{\mathrm{a}}$ \\
Espermios inmóviles (\%) & $7.35 \pm 0.84^{\mathrm{a}}$ & $5.73 \pm 0.94^{\mathrm{a}}$ & $6.33 \pm 0.99^{\mathrm{a}}$ \\
\hline
\end{tabular}

Superíndices diferentes dentro de filas indican diferencias estadísticas significativas $(p<0.05)$ 
Cuadro 2. Efecto de la adición de de ciclodextrinas cargadas de colesterol (CLC) sobre las características microscópicas de semen pos-descongelación de toros Holsteir Friesian (media \pm error estándar)

\begin{tabular}{lccc}
\hline & $\begin{array}{c}\text { mg CLC } \\
\text { (T1) }\end{array}$ & $\begin{array}{c}1.5 \mathrm{mg} \text { CLC } \\
(\mathrm{T} 2)\end{array}$ & $\begin{array}{c}2 \mathrm{mg} \text { CLC } \\
(\mathrm{T} 3)\end{array}$ \\
\hline Motilidad total (\%) & $67.73 \pm 0.7^{\mathrm{a}}$ & $79.59 \pm 0.77^{\mathrm{b}}$ & $74.73 \pm 0.79^{\mathrm{c}}$ \\
Motilidad progresiva (\%) & $54.73 \pm 0.72^{\mathrm{a}}$ & $63.42 \pm 0.83^{\mathrm{b}}$ & $55.83 \pm 0.85^{\mathrm{a}}$ \\
Vitalidad (\%) & $63.26 \pm 0.68^{\mathrm{a}}$ & $75.84 \pm 0.76^{\mathrm{b}}$ & $70.53 \pm 0.87^{\mathrm{c}}$ \\
Host (\%) & $46.89 \pm 0.59^{\mathrm{a}}$ & $60.43 \pm 0.6^{\mathrm{b}}$ & $55.06 \pm 0.63^{\mathrm{c}}$ \\
Morfología (\%) & $5.14 \pm 0.16^{\mathrm{a}}$ & $4.54 \pm 0.15^{\mathrm{b}}$ & $4.75 \pm 0.15 \mathrm{~b}$ \\
Espermios inmóviles (\%) & $32.27 \pm 0.7^{\mathrm{a}}$ & $20.41 \pm 0.77^{\mathrm{b}}$ & $25.27 \pm 0.79^{\mathrm{c}}$ \\
\hline
\end{tabular}

Superíndices diferentes dentro de filas indican diferencias estadísticas significativas $(p<0.05)$

to pos-descongelación del semen (Moce y Graham, 2014).

La motilidad total inicial fue superior a la reportada por Bach (2009) de $67.9 \%$ con 0 y 2 mg de CLC, posiblemente debido a que el esperma tuvo un mayor tiempo de estabilización (18 horas) durante la fase de refrigeración. La motilidad progresiva tampoco presentó diferencias significativas entre tratamientos; por otro lado, Rajoriya et al. (2014) trabajando con búfalos obtuvieron motilidades progresivas de 76.1 y $77.6 \%$ utilizando 0 y 2 mg de CLC.

No se encontró diferencia significativa entre tratamientos para la prueba de vitalidad espermática, resultado similar al reportado por Rajoriya et al. (2014), aunque con valores inferiores con $0 \mathrm{mg}$ de CLC $(80.6 \%)$ y con $2 \mathrm{mg}$ de CLC (82.0\%). Tampoco hubo diferencia significativa en la prueba de integridad de membrana citoplasmática entre tratamientos. Por otro lado, Madrid-Bury et al. (2005) reportaron valores Host entre 70.2 y $73.5 \%$.

Los valores de espermatozoides anormales fueron de 5.0, 4.6 y $4.9 \%$ para $\mathrm{T} 1$, $\mathrm{T} 2$ y T3, respectivamente, no existiendo diferencias significativas entre tratamientos; no obs- tante, fueron valores inferiores al 10.3 y $9.7 \%$ reportados por Mejía (2017) y Ramonez (2013), respectivamente, en semen refrigerado. Tampoco se registraron diferencias significativas entre tratamientos en la variable de espermios inmóviles (Cuadro 1); resultados que difieren con Gallardo y Vargas (2015), quienes evaluaron semen bovino en la etapa de precongelación utilizando los dilutores AndroMed (Minitube), BioXcell (MV Technologies) y Triladyl (Minitube) obteniendo 17,25 y $32 \%$ de espermatozoides inmóviles, respectivamente.

\section{Efecto del CLC sobre el Semen Pos- Descongelación}

T2 presentó una mayor motilidad que T3 y T1 ( $<<0.05$; Cuadro 2), logrando una diferencia porcentual de $11.86 \%$ frente a $\mathrm{T} 1$; lo cual concuerda con Mocé et al. (2014), quienes obtuvieron $78.4 \%$ de motilidad total utilizando $2 \mathrm{mg}$ de CLC frente al control con $75.2 \%$. Otros autores obtuvieron motilidades más bajas que el presente estudio, aunque siempre superiores a los tratamientos control (Purdy y Graham, 2004; Moraes et al., 2010; Amorim et al., 2009). Así mismo, Mocé y Graham (2006) no encontraron diferencias significativas entre el control y el tratamiento con $2 \mathrm{mg}$ de CLC (58\% de motilidad total). 
Estos resultados permiten indicar que los tratamientos con CLC en el semen fresco redujeron el daño del esperma producido por los cambios de temperatura durante la criopreservación, posiblemente debido a una mayor estabilización de la membrana plasmática, producto del ingreso de colesterol a la misma (Purdy y Graham, 2004a; Moore et al., 2005; Mocé et al., 2010). La concentración más utilizada en los trabajos revisados en la literatura científica se sitúa entre 1 y $2.5 \mathrm{mg}$ para $120 \mathrm{x} 10 \mathrm{v}$ espermatozoides $/ \mathrm{ml}$, correlacionándose negativamente con la fertilidad en concentraciones inferiores a $0.5 \mathrm{mg}$ o superiores a $4 \mathrm{mg}$ de CLC (Mocé et al., 2010).

Se observaron diferencias significativas entre T1 y T2 y entre T2 y T3 en motilidad progresiva ( $p<0.05$; Cuadro 2). Datos similares fueron reportados por Rajoriya et al. (2014) con $66.4 \%$ para el tratamiento con $2 \mathrm{mg}$ de CLC y de $5.9 \%$ para el control, y muy superiores al 32\% reportado por Mocé et al. (2014) utilizando $2 \mathrm{mg}$ de CLC, al $18 \mathrm{y}$ $6 \%$ con $2 \mathrm{mg}$ y $4 \mathrm{mg}$ de CLC reportado por Moce y Graham (2014) y al 16\% reportado por Moraes et al. (2010) utilizando $1.5 \mathrm{mg}$ de CLC. Catena y Cabodevila (1999) indican que el semen de buena calidad recientemente descongelado presenta una motilidad progresiva de $40-50 \%$, mientras que Awad y Graham (2002) indican que con el tratamiento con CLC se obtiene una mayor proporción de espermatozoides viables y progresivamente móviles después de la criopreservación. No obstante, con base a los resultados obtenidos, se deduce que a mayor cantidad de CLC por tratamiento, la motilidad total y progresiva tiende a disminuir, posiblemente debido a la saturación de la membrana plasmática con colesterol, lo cual afectaría su normal funcionamiento (Purdy y Graham, 2004b).

La viabilidad espermática fue significativamente diferente entre los tres tratamientos, siendo mejor en T2 $(75.84 \%)(\mathrm{p}<0.05)$. Otros autores indican una mayor vitalidad (7274\%) utilizando $2 \mathrm{mg}$ de CLC (Mocé et al.,
2014; Rajoriya et al., 2014). Así mismo, Mocé y Graham (2014) encontraron una viabilidad espermática en la descongelación de $38 \%$ en el tratamiento control y de 61 y $48 \%$ con 2 mg y $4 \mathrm{mg}$ de CLC, respectivamente, mientras que Bach (2009) obtuvo una viabilidad de 48.4 y $50.4 \%$ para el grupo control y el de $2 \mathrm{mg}$ de CLC, respectivamente. La disminución encontrada en este estudio fue, sin embargo, menor a la reportado por Thomas et al. (1998), quienes afirman que se produce una disminución del $50 \%$ de viabilidad espermática durante el proceso de criopreservación, debido principalmente al efecto de la temperatura y la presión osmótica, que ocasionan cambios en la permeabilidad, composición lipídica de las membranas espermáticas y en el líquido intracelular. Esto permite suponer que los espermatozoides tratados con CLC tienen una mayor tolerancia a la criopreservación, ya que aumentan su ratio colesterol: fosfolípidos, producto del ingreso de colesterol a la membrana, volviéndose resistentes a los efectos de la transición de fase lipídica causado por el shock térmico.

La reacción a la prueba de endosmosis (HOST) indicó diferencias significativas entre tratamientos ( $<<0.05$; Cuadro 2), en concordancia con resultados reportados por otros autores (Purdy y Graham, 2004; Amorim et al., 2009), mientras que Mocé y Graham (2006) obtuvieron una integridad de membrana de $62 \%$ con $2 \mathrm{mg}$ de CLC y Moncayo (2016) reportó valores de 68.7 y $61.8 \%$ para toros Holstein utilizando los dilutores Triladyl y Andromed, respectivamente. No obstante, Purdy et al. (2005) obtuvieron mayores valores HOST ( $76 \%$ con $1.5 \mathrm{mg}$ de CLC y $60 \%$ para el grupo control). Las membranas íntegras permiten la capacitación espermática, la penetración de los revestimientos del ovocito, la unión con la zona pelúcida y la fusión de la membrana (Rodríguez et al., 1997). Las membranas celulares son las estructuras celulares que sufren mayor daño en los procesos de congelación, debido a la pérdida de fluidez de sus componentes lipídicos (Parks y Graham, 1992). 
Hubo diferencia significativa en la morfología espermática entre T1 y T2 y entre T1 y T3 ( $<<0.05$; Cuadro 2). Palacios (2015), por otro lado, encontró una mayor presencia de anormalidades (11\%). Los resultados obtenidos indican que la inclusión de CLC en el semen no tuvo relación directa con el porcentaje de anormalidades presentes en el esperma.

Por último, se observó diferencia significativa entre tratamientos con relación a los porcentajes de espermios inmóviles $(\mathrm{p}<0.05$; Cuadro 2). Por otro lado, Mejía (2017) reportó un porcentaje de inmovilidad extremo del $60.8 \%$. El valor de esperma inmóvil se relaciona directamente con el porcentaje de motilidad total $(79.59 \%)$

\section{Conclusiones}

La adición de metil- $\beta$-ciclodextrina cargada de colesterol previa a la congelación del semen bovino mejoró todos los parámetros espermáticos pos-descongelación (motilidad total, motilidad progresiva, vitalidad e integridad de membrana), con excepción de la morfología.

- Los mejores resultados se observaron con la adición de $1.5 \mathrm{mg}$ de CLC en 120 millones de espermatozoides $/ \mathrm{ml}$ ).

\section{Literatura Citada}

1. Amorim EA, Graham JK, Spizziri B, Meyers M, Torres CA. 2009. Effect of cholesterol or cholesteryl conjugates on the cryosurvival of bull sperm. Cryobiology 58: 210-214. doi: 10.1016/ j.cryobiol.2008.12.007

2. Awad MM, Graham JK. 2002. Effect of adding cholesterol to bovine spermatozoa on motility parameters and cell viability after cryopreservation. Cryobiology 45: 256-257.
3. Bach KD. 2009. Effects of cholesterol on bovine sperm survival during cooling $\&$ freezing. MSci Thesis. USA: Cornell University. $84 \mathrm{p}$.

4. Catena M, Cabodevila J. 1999. Evaluación de semen bovino congelado. En: Simposio Internacional de Reproducción Bovina (UNCPBA). Tandil, Argentina.

5. Correa JR, Zavos PM. 1994. The hipoosmotic swelling test: its employment as an assay to evaluate the functional integrity of the frozen-thawed bovine sperm membrane. Theriogenology 42: 351-360. doi: 10.1016/0093-691X(94)90280-1

6. Gallardo J, Vargas C. 2015. Evaluación de tres diluyentes para criopreservar semen bovino de toros cruce Sahiwal (Bos taurus) en el trópico húmedo. Tesis de Ingeniero Agropecuario. Santo Domingo de los Tsáchilas, Ecuador: Univ. de las Fuerzas Armadas. 70 p.

7. Hummersted RH, Graham JK, Nolan JP. 1990. Cryopreservation of mammaliam sperm: what we ask them to survive. J Androl 11: 73-88. doi: 10.1002/j.19394640.1990.tb01583.x

8. Madrid-Bury N. 2005. ¿Es posible predecir la fertilidad en los toros? En: González-Stagnaro C, Soto-Belloso E (eds). Manual de ganadería doble propósito. Maracaibo, Venezuela: Astro Data. p 631-635.

9. Mejía E. 2017. Evaluación pre y post congelación del semen obtenido con vagina artificial y electroeyaculador en el ganado criollo. Tesis de Magíster. Cuenca, Ecuador: Univ. de Cuenca. 54 p.

10. Mocé E, Graham JK. 2006. Cholesterol-loaded cyclodextrins added to fresh bull ejaculates improve sperm cryosurvival. J Anim Sci 84: 826-833. doi: 10.2527/2006.844826x

11. Mocé E, Purdy PH, Graham JK. 2010. Treating ram sperm with cholesterol loaded cyclodextrins improves cryosurvival. Anim Reprod Sci 
118:236-247. doi: 10.1016/j.anire-prosci.2009.06 .013

12. Mocé E, Graham J.K. 2014. Cholesterol-loaded cyclodextrins added to fresh bull ejaculatesimprove sperm cryosurvival. J Anim Sci 84: 826-833.

13. Mocé E, Tomás C, Blanch E, Graham JK. 2014. Effect of cholesterol-loaded cyclodextrins on bull and goat sperm processed with fast or slow cryopreservation protocols. Animal 8: 771-776. doi: 10.1017/S1751731114000226

14. Moore AI, Squires EL, Graham JK. 2005. Adding cholesterol to the stallion sperm plasma membrane improves cryosurvival. Criobiology 51:241-249. doi: 10.1016/j.cryobiol.2005.07.004

15. Moncayo S. 2016. Evaluación de la calidad seminal de reproductores bovinos antes y después del proceso de criopreservación. Tesis de Ingeniero en Biotecnología de los Recursos Naturales. Quito, Ecuador: Univ. Politécnica Salesiana. 69 p.

16. Moraes EA, Graham JK, Torres CA, Meyers M, Spizziri B. 2010. Delivering cholesterol or cholestanol to bull sperm membranes improves cryosurvival. Anim Reprod Sci 118: 148-154. doi: 10.1016/ j.anireprosci.2009.08.002

17. Palacios L. 2015. Evaluación de dos diluyentes caseros y un diluyente comercial para criopreservar semen bovino de las razas Brown Swiss y Patua (Bos taurus) en el trópico húmedo. Tesis de Ingeniero Agropecuario. Santo Domingo, Ecuador: Univ. de las Fuerzas Armadas. $90 \mathrm{p}$.

18. Parks J, Graham J. 1992. Effects of cryopreservation procedures on sperm membranes. Theriogenology 38: 209-222. doi: 10.1016/0093-691x(92)90231-f
19. Purdy PH, Graham JK. 2004. Eûect of cholesterol-loaded cyclodextrin on the cryosurvival of bull sperm. Cryobiology 48: 36-45. doi: 10.1016/j.cryobiol.2003.12.001

20. Purdy PH, Graham JK. 2004. Effect of cholesterol to bull sperm membranes on sperm capacitation, the acrosome reaction, and fertility. Biol Reprod 71: 522-527. doi: 10.1095/biolreprod.103.025577

21. Purdy PH, Fox MH, Graham JK. 2005. The fluidity of Chinese hamster ovary cell and bull sperm membranes after cholesterol addition. Cryobiology 51: 102-112. doi: 10.1016/j.cryobiol.2005.05.004

22. Rajoriya J, Prasad J, Ghosh S, Snehal S, Ramteke N, Barik G, Das Y, et al. 2014. Cholesterol loaded cyclodextrin increases freezability of buffalo bull (Bubalus bubalis) spermatozoa by increasing cholesterol to phospholipid ratio. Vet World 7: 702-706. doi: 10.14202/ vetworld.2014.702-706

23. Ramónez J. 2013. Evaluación de dos agentes crioprotectores no permeables y un diluyente comercial (triladyl) en la congelación de semen bovino. Tesis de Magíster. Cuenca, Ecuador: Univ. de Cuenca. $89 \mathrm{p}$.

24. Rodríguez, $H$, Larsson B, Perstoft $H$. 1997. Evaluation of sperm damage and techniques for sperm clean-up. Reprod Fert Develop 9: 297-308. doi: 10.1071/ r96081

25. Thomas CA, Garner DL, DeJarnette JM, Marshall CE. 1998. Effect of cryopreservation of bovine sperm organelle function and viability as determined by flow cytometry. Biol Reprod 58: 786-793. doi: 10.1095/ biolreprod58.3.786 